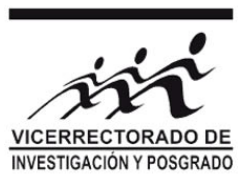

\title{
Mujeres físicas de pregrado y posgrado: un análisis estadístico comparativo
}

\author{
María Luisa Cerón Loayza ${ }^{*}$, Mirian E. Mejía Santillán ${ }^{1}$, Silvia M. Espinoza Suarez ${ }^{2,3}$, Fanny E. Mori \\ Escobar $^{1}$, Mabel E. Tesillo Quispe ${ }^{1}$ y María C. Trujillo Saenz ${ }^{1}$ \\ ${ }^{1}$ Universidad Nacional Mayor de San Marcos, Lima, Perú \\ ${ }^{2}$ Grupo de Investigación de Materias Funcionales - UNMSM, Lima, Perú \\ ${ }^{3}$ TECSUP, Lima, Perú
}

Recibido 02 octubre 2019 - Aceptado 30 diciembre 2019

\begin{abstract}
Resumen
Un análisis estadístico comparativo realizado en la Facultad de Ciencias Físicas de la Universidad Nacional Mayor de San Marcos (UNMSM) nos permite visibilizar que existe una desproporción contundente entre el número de estudiantes ingresantes mujeres con los ingresantes varones a nivel del Posgrado. Así mismo, se observa una situación similar entre el número de estudiantes ingresantes y egresadas mujeres a nivel de Pregrado. Estos resultados basados en los datos estadísticos verifican que este campo de la ciencia sigue siendo masculinizado.
\end{abstract}

Palabras clave: Mujeres físicas del Perú, mujeres físicas de pregrado y postgrado, análisis estadístico.

\section{Undergraduate and graduate physicist women: a comparative statistical analysis}

\begin{abstract}
A comparative statistical analysis carried out at the Faculty of Physical Sciences, UNMSM, allows realize that there exists a marked disproportion between the numbers of admitted female and male students at the graduate level. Likewise, a similar situation is observed between the numbers of admitted and graduated female undergraduate students. These results based on statistical data verify that this field of science continues to be masculinized.
\end{abstract}

Keywords: Physical women of Peru, undergraduate and postgraduate physical women, statistical analysis.

\section{Introducción}

De acuerdo con un estudio reciente de la Organización de Naciones Unidas (ONU), realizado en 14 países, la probabilidad de que una mujer obtenga una maestría o un doctorado es de $8 \% \circ 2 \%$, respectivamente, mientras que para un hombre las opciones son más del doble en cada caso. Existen datos estadísticos de países latinoamericanos con respecto a la participación de las Mujeres en el campo de las CTIM (Ciencias, Tecnología, Ingenierías y Matemáticas) pero puntualmente la carrera de Ciencias Fiscas, no. En la Figura 1 observamos el porcentaje de mujeres investigadoras en países de América Latina y el Caribe (Fuente: Instituto de Estadística de la Unesco y Red de indicadores de Ciencia y Tecnología Iberoamericano e Interamericana, RICYT), se puede observar que Perú, no aparece hasta esa fecha registrada; sin embargo, informes del I Censo Nacional de Investigación y Desa-

\footnotetext{
${ }^{*}$ mceronl@unmsm.edu.pe
}

rrollo, CONCYTEC Dirección de Investigación y Estudios (2015), se tiene como datos comparativos entre los investigadores mujeres y varones al 100 por ciento, que el 68.1 por ciento del total fueron hombres (2,298 investigadores) y 31.9 por ciento fueron mujeres (1,074 investigadoras). Lo que que significa que en el Perú se puede observar que por cada investigadora hay 2.1 investigadores. ¿Y cuántas investigadoras del total serán físicas?

Datos más puntuales, para Argentina, según el Instituto de Estadística de la UNESCO (2012), el porcentaje de mujeres científicas es del 62 por ciento; para Chile existe un $14 \%(14,4 \%$ en $1995 ; 13,6 \%$ en 2018$)$ (Fuente: SOCHEFI, Sociedad Chilena de Física).

A partir de estos datos estadísticos mostrados es necesario tener una estadística cuantitativa de mujeres físicas, y sobre todo saber cuánto es la población de mujeres en nuestra institución, por ello en este trabajo se presenta un análisis comparativo entre mujeres y varones ingresantes 
y egresadas en Pre y Posgrado de la Facultad de Ciencias Físicas (FCF) de la Universidad Nacional Mayor de San Marcos (UNMSM). Parte del trabajo fue presentado en el Second IUPAP Conference on Women in Physics en el año 2005, realizado en Río de Janeiro [Cer05], [Cer08] y los resultados de este análisis fueron una de las principales motivaciones para discutir la poca participación de las mujeres en la FCF.

Estas conferencias a nivel mundial y en especial en Latinoamérica son de mucho interés, permiten compartir esencialmente un tema en común: la discriminación y la poca participación de las mujeres en Física. A pesar de las diferencias en cultura, etnias y religión que puedan existir; existen muchas experiencias en común que comparten las mujeres físicas. Asimismo, también hay espacios para discutir sobre la investigación que cada una desarrolla, por ello se busca analizar y discutirlos sobre los datos estadísticos que presenta cada región sobre este tema en Física. Para nuestra región nos planteamos cuáles son las razones por las que las mujeres no optan por un carrera en ciencias, en especial la Física en pregrado? Y de las que se animan a estudiar esta profesión, ¿por qué algunas deciden abandonar la carrera durante sus estudios, sin culminarla?

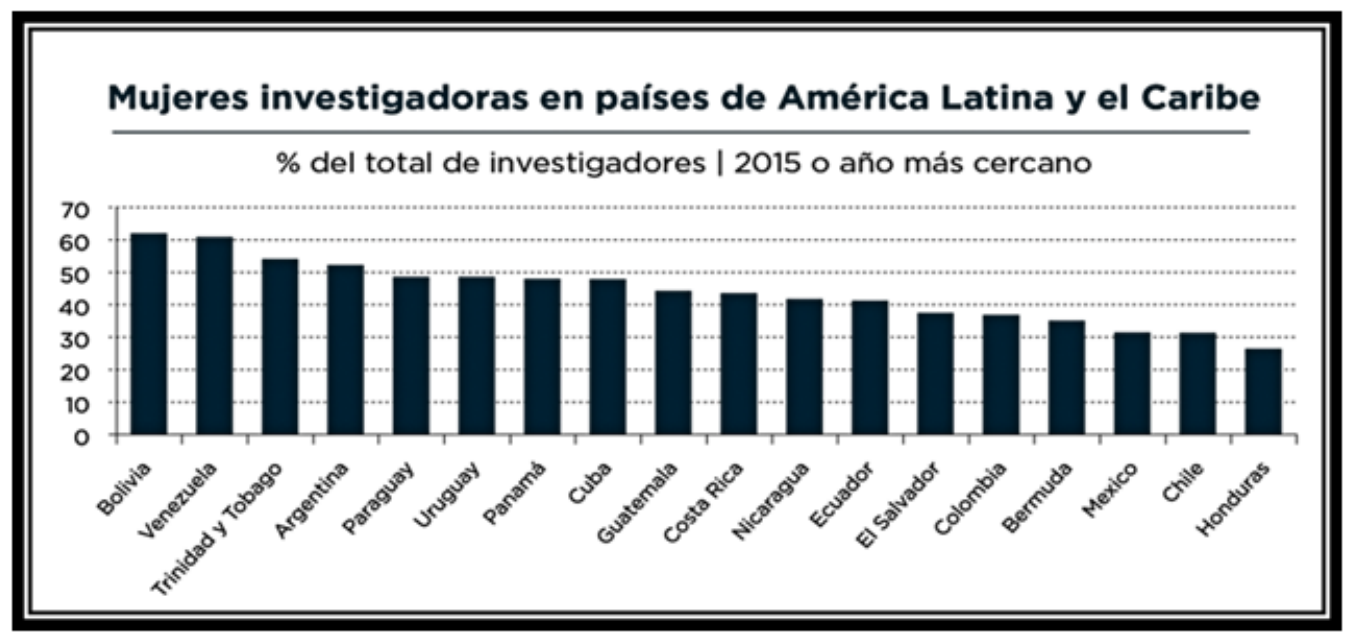

Figura 1: Mujeres investigadoras en países de América Latina y el Caribe (Fuente: Instituto de Estadística de la Unesco y Red de indicadores de Ciencia y Tecnología Iberoamericano e Interamericana, RICYT).

\section{Datos estadísticos de ingresantes y egresa- das}

Se muestran datos estadísticos comparando cifras de estudiantes mujeres y varones de Pre y Posgrado de la Facultad de Ciencias Físicas, donde se puede visualizar que existe una desproporción entre las estudiantes ingresantes mujeres en comparación con los ingresantes varones y en una proporción menor aún están las egresadas en pregrado. Se ha entrevistado a algunas de ellas, dado que son escasas, y muchas abandonan la carrera por diversas razones: trabajo, problemas familiares o simplemente porque no es la carrera que desean, generando que se trasladen a otras facultades vía un examen externo o interno.

Habría que preguntarnos, si hay suficiente motivación desde los colegios para que las mujeres opten por una carrera profesional en las Ciencias Físicas? y si tanto el varón como la mujer interesadas en las áreas de ciencias, en especial en las Ciencias Físicas, tienen las mismas oportunidades en el campo laboral? Ante esta situación hay que plantear posibles estrategias de solución para incrementar la participación de la mujer en el campo de la Física.

En la Figura 2 podemos observar el número de estudiantes ingresantes mujeres de pregrado desde el año 1997 hasta 2008. A continuación se presenta una interpretación de los datos:

\section{Interpretación}

a) 53 mujeres, que representan el 50 por ciento de las 106 de mujeres que ingresaron a la EAP de Física desde 1997 al 2008, se encuentran estudiando.

b) 37 mujeres, que representan el 34.91 por ciento de mujeres del total que ingresaron, han abandonado la carrera.

c) 13 mujeres, que representan el 12.26 por ciento del total de mujeres, egresaron.

d) 3 mujeres, que representan el 2.83 por ciento del total de mujeres, efectuaron su reserva de matrícula.

Son muy preocupantes estos resultados mostrados en la Figura 1 que recoge datos de 11 años, en que sólo egresaron 13 mujeres físicas a esa fecha registrada. 


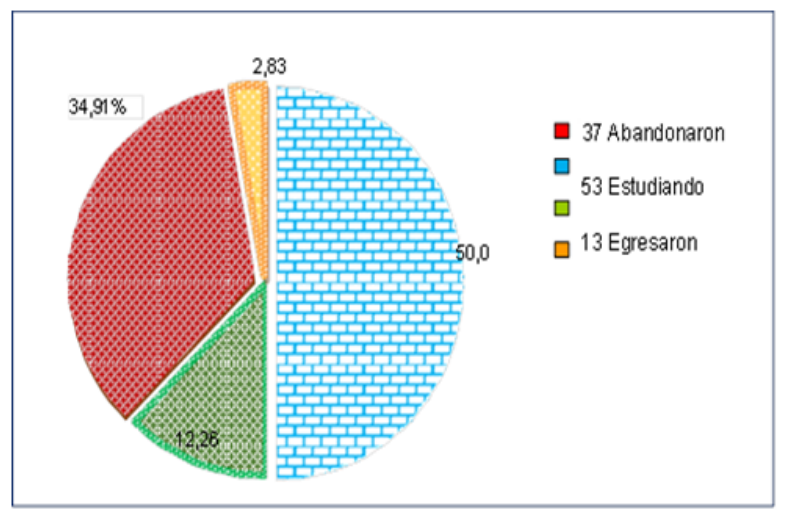

Figura 2: Número de estudiantes ingresantes mujeres de pregrado desde el año 1997 hasta 2008.

En el caso del Posgrado, los números de ingresantes y de egresadas se pueden observar en las Figuras 2 y 3 Considerando que el Programa de Maestría se desarrolla en un periodo de dos años, incluyendo la tesis de investigación; habría que preguntarnos, cuántas siguen un posgrado? Cuántas logran culminar y obtener el grado? Existe una serie de preguntas que requieren respuestas para mejorar el desarrollo profesional de las Mujeres Físicas. El desempeño de la Mujer Física como investigadora es esencial en nuestra sociedad, muestra un ejemplo de mentoria en las estudiantes en formación, en nuestra facultad de ciencias físicas de la UNMSM tenemos muy buenos ejemplos de dos mujeres físicas, que ya no se encuentran entre nosotras, tal es el caso de la primera Astrónoma del Perú, María Luisa Aguilar (1938-2015), quien en 1981 fundó el Seminario de Astronomía y Astrofísica, el que, desde el 2001, pasa a denominarse Seminario Permanente de Astronomía y Ciencias Espaciales - SPACE, semillero de futuras generaciones de profesionales en astronomía [Wik19], y la Dra Mariella Jeanette Berrocal Tito (1963-2014), quien empezaba a formar un Grupo consolidado en la investigación. Así mismo en las pocas universidades dedicadas a las carreras básicas fuertes, como la Ciencias Físicas, tenemos otro buen ejemplo en la Universidad Nacional de Ingeniería, la Dra. Mónica Gómez León, quien recibió el Premio L'Oreal el año 2011.

En la Figura 3.a), se observa el número de estudiantes ingresantes por año, desde el año 1991 hasta 2005; al Programa de Posgrado; mientras que en la Figura 3(b) se observa el número de egresadas por año, es decir, el número de mujeres que lograron culminar los estudios de posgrado desde 1991 hasta el 2006. De manera particular tenemos reportado lo siguiente: una docente de la Facultad que ingresó al programa de maestría en el año 2000, como se observa en la Figura 3. a), culminó sus estudios en el año 2004. Dos docentes que ingresaron en el año 2002, culminaron sus estudios en el año 2004. Por tanto, se observa en la Figura 3(b), para el año 2004, las 3 egresadas a nivel de posgrado.

En los 15 años (1991-2005) a nivel de posgrado sólo se registran 19 ingresantes mujeres físicas y de ellas solo 4 culminaron sus estudios, sin las obtenciones del grado de magíster (datos al 2005). No son cifras alentadoras; por el contrario son resultados que se deben considerar para optimizar y alentar a la plana de mujeres físicas de la institución a motivarlas a una mayor participación.
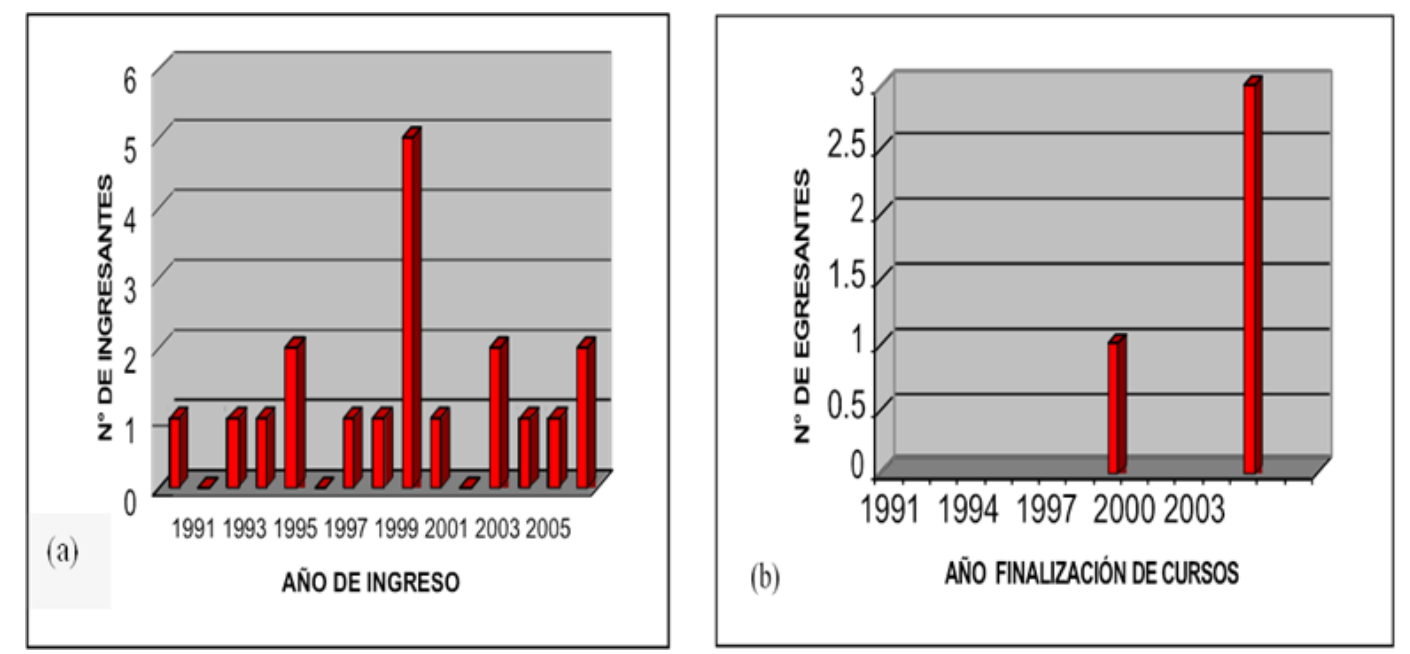

Figura 3: Estudiantes mujeres de posgrado: (a) Número de estudiantes ingresantes desde el año 1991 hasta 2005 a nivel de Posgrado; (b) Número de egresadas a nivel posgrado. 
En la Figura 4 se observan los datos comparativos entre ingresantes mujeres y varones desde el año 1991 hasta el 2008. Podemos analizar que de los años 1993 y 1994 sólo una ingresante se animó a participar del programa de Posgrado; en el año 1997 y 1998 sucedió algo similar. En el 2005 la diferencia es abismal, son 25 varones ingresantes y solo 2 ingresantes mujeres; con estas estadísticas mostra- das sería propicio preguntarnos cuantas de las mujeres que ingresaron lograron culminar sus estudio de Posgrado? Por ejemplo; si en el año 1991 ingresó una estudiante, debería egresar el año 1993, y en el cuadro de la Figura 3(b), no se observa este resultado, y así podríamos analizar cada año.

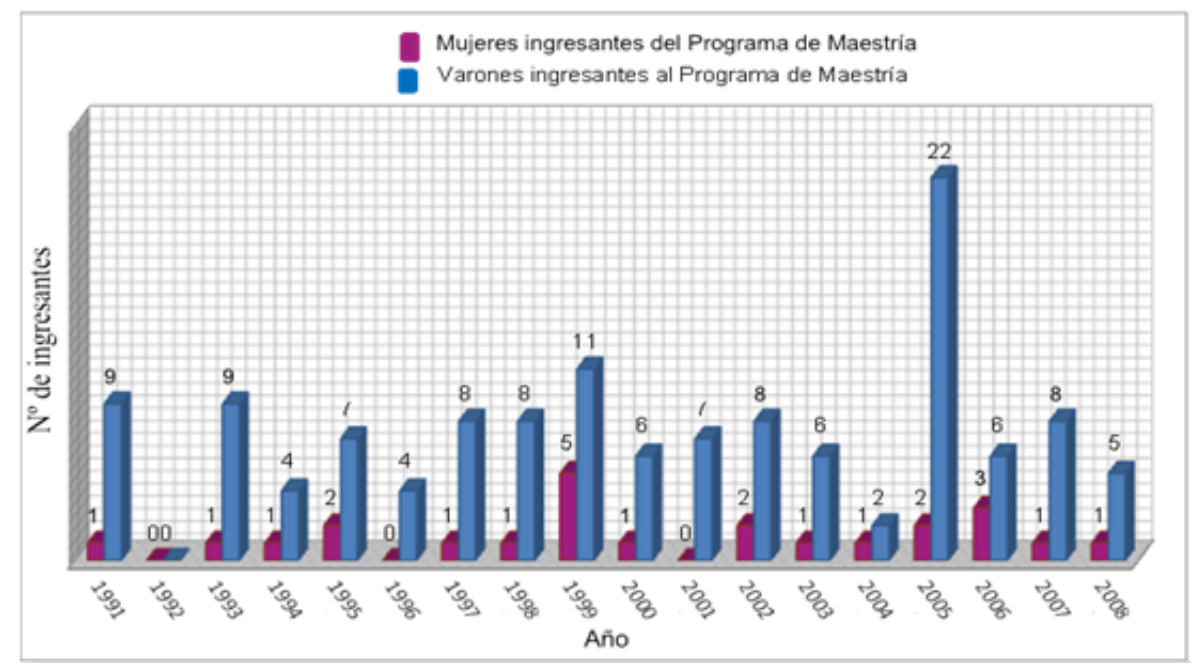

Figura 4: Datos comparativos entre ingresantes mujeres y varones del Programa de la Maestría en física desde el año 1991 hasta el 2008.

\section{Posibles obstáculos}

Existen muchas razones que obstaculizan el interés de continuar con la carrera de ciencias físicas. Primero en una etapa inicial de pre grado, luego cuando se concluyen los estudios y se espera continuar con el posgrado y seguir para la consolidación como investigadoras. Así podríamos definir que las niñas desde el colegio, no se motivan por una profesión en ciencias, porque muchas veces es la propia familia, profesores, compañeros y la sociedad en general que generan estereotipos de género, donde carreras como las ciencias son solo para varones. Existe un desconocimiento de lo que hace la mujer física y de cómo puede contribuir a la sociedad. Ahora como estudiantes de pregrado muchas mujeres abandonan la carrera por razones económicas, otras porque se trasladan a otras carreras. En cuanto a las mujeres profesionales físicas, podríamos puntualizar algunas de las razones y agruparlas de la manera siguiente:

1. El trabajo como profesionales (por ejemplo en Perú cuando se terminan los estudios de pregrado o desde los últimos ciclos de los estudios universitarios, se realizan prácticas pre profesionales; si hay oportunidad se logrará hacerlo en la Facultad como catedrático o en alguna otra institución para poder solventarse económicamente ya que la responsabilidad muchas veces aumenta en for- ma directamente proporcional con el paso de los años. En otros casos, se buscan becas para continuar con el avance académico).

2. La familia (cuidado de las niñas y de los niños, el cuidado de los padres) y

\section{La investigación.}

Estos tres puntos mencionados sobre las actividades de las mujeres físicas se desarrollan en forma paralela. Razón por la cual, su tiempo debe ser distribuido y no hay una dedicación en su totalidad en la investigación. Debemos considerar que los reportes demuestran que tener una familia no debe ser un factor limitante para la participación de la mujer [Don02] |lvi02], las mujeres sin niños no son más exitosas que la mujer que tiene niños. Otra razón principal es la falta de apoyo, pues se requiere inversión en una tesis de maestría y/o doctorado, lo ideal sería que se apoye con becas a mujeres que apostaran por un posgrado en ciencias, que se brinde un porcentaje mínimo para aumentar la cuota de género en ciencias, de esa forma aumentaría su producción, dado que brinda una tranquilidad económica sin afectar su calidad de vida. Este tipo de financiación aparentemente recién se está llevando a cabo, para mujeres y varones por igual, tal como en la Universidad Nacional Mayor de San Marcos (2013-2015), donde se considera el límite de edad, que podría ser discrimina- 
toria para muchas. Sería importante e interesante poder invocar a otras entidades de apoyo para que más jóvenes tengan mayor oportunidad de aceptación. Por ello, es preciso visibilizar nuestros propios logros. Asimismo, se debe concientizar a nuestros colegas varones para fomentar igualdad de oportunidades, es necesario plantear estrategias para poder generar un mayor interés en las carreras de las Ciencias en general y de la Física en especial.

Se tiene que recalcar que desde el año 1998 la mayoría de las mujeres físicas de la Facultad de Ciencias Físicas de la UNMSM (que han realizado un posgrado en el extranjero) lo han hecho en el Brasil. Por ejemplo, en el año 2001 tres estudiantes mujeres se fueron a estudiar un posgrado. Asimismo, en nuestra Facultad de Ciencias Físicas de la UNMSM se cuenta sólo con una especialista con nivel de doctorado a la fecha (considerando que dos profesoras investigadoras con grado de doctoras han fallecido). Este es un problema que hay que afrontar y posibilitar mayor oportunidad, divulgando los centros de investigación que ofrecen becas, las cuales permitirán a las estudiantes tomar la firme decisión de continuar.

\section{Conclusiones}

Existe un resultado muy preocupante con los datos mostrados tanto entre las mujeres egresadas de pregrado como de posgrado. Donde solo 13 de un total de 106 mujeres físicas egresaron del pregrado en 11 años (19972008) y solo 4 de 19 mujeres físicas egresaron del posgrado en 15 años, sin considerar la obtención del grado de magíster.

Los países de Latinoamérica y otros países comparten problemas en común: la poca participación de las mujeres en la Física. Se busca planes de trabajo: primero convocar a todas las Mujeres Físicos del Perú de las diferentes universidades de la región, ya sean universidades públicas o privadas que ofrezcan la carrera de Ciencias Físicas, para tener entes de comunicación entre estudiantes de pregrado y posgrado y nombrar representantes internas, que se comprometan a trabajar y concientizar esta labor.

Se debe concientizar a las autoridades y colegas de las diferentes instituciones para consolidar las vocaciones de las estudiantes físicas y romper todas las brechas de género para lograr una mayor y mejor participación.

\section{Agradecimientos}

Las autoras agradecen la colaboración de la exSecretaria de la Unidad de Posgrado, Janet Ascensio por el apoyo en el acceso a los archivos académicos de los ingresantes y egresados a nivel de pregrado y posgrado (trabajadora administrativa hasta el 2017). Asimismo, al psicólogo Sandro Lazaro, por su apoyo con el manejo de datos de la Facultad de Ciencias Físicas - UNMSM.

\section{Referencias}

[Cer05] Cerón, M. (2005). Preliminary Analysis of Female Physics Students at the Greater National University of San Marcos, Peru. AIP conference proceedings, 795, 147-148.

[Cer08] Cerón, M., Huaypar, Y. and Bravo, J. (2008). Women in Physics in Perú. AIP conference proceedings, $1119,153-154$

[Don02] Li, D. and Karplus, B. (2002). Learning from the World. AIP Conference Proceeding, "Women in Phy- sics: The IUPAP International Conference on Women in Physics". 628, 3.

[Ivi02] Ivie, R., Czujko, R. and Stowe, K. (2002). The International Survey of Women in Physics. In Women Physicists Speak The 2001 International Study of Women in Physics (5-17). United States: AIP Publishing.

[Wik19] Wikimedia, Inc. (2019). Sitio web de María Luisa Aguilar: https://es.wikipedia.org/wiki/ Mar\%C3\%ADa_Luisa_Aguilar 
